\title{
THE DEFINITIONAL POWER OF WORDS
}

\author{
Branimir K. Boguraev \\ Computer Laboratory \\ University of Cambridge \\ Corn Exchange Street \\ Cambridge CB2 3QG, England
}

I am deliberate in introducing ambiguity in the title. Part of my thesis in this brief note is going to be that there is a wealth of information relevant to a range of natural language processing functions available and extractable from the definitions of words found in obvious places like dictionaries. By dictionaries here I mean monolingual dictionaries of the style exemplified by, e.g., The Longman Dictionary of Contemporary English, The Collins English Dictionary, or Webster's Seventh New Collegiate Dictionary. This is hardly surprising, given that what is to be found in a dictionary is essentially the result of a substantial amount of work on analysing and collating data about real language and elicitating collocational and distributional properties of words and applying certain common principles of defining their meaning. Furthermore, I am going to argue that carefully exploited interplay between notions of "words" and "primitives", can add substantial leverage to the functionality and coverage of a natural language processing system.

Several factors and related phenomena underlie the current interest in words, and consequently word resources. Over the last decade there has been the emergence of theories of grammar and grammatical frameworks (e.g., Lexical Functional Grammar, Generalized Phrase Structure Grammar, PATR-II, Functional Unification Grammar, Lexicon Grammar, Word Grammar) placing heavy emphasis on the lexicon, where elaborate information about the grammatical and logical idiosyncracies of words is stored and used to drive various parsing systems. More relevant to this panel, however, is the progress in both the practical aspects of natural language processing (various techniques for e.g., performing text analysis or building and customising natural language interfaces) and the theoretical issues of knowledge representation and access.

It would not be too provocative to state that the current understanding of how to go about building practical systems is sufficient to make such a task tractable. However, realistic natural language processing programs fall in the general class of knowledge-based systems in AI, and they all require significant amounts of structured knowledge about the real world, as well as about a particular domain of discourse. There are typically two problems here, one related to the scaling up of a prototype by expanding its knowledge base and the other related to the activity of transporting or customising an existing system. In both cases the real culprit is the knowledge acquisition bottleneck. Given the availability online of suitable machine readable resources, namely dictionaries and encyclopaedias, there is strong hope that some model of the common world may be localised and extracted from such sources. Even if individual applications may require additional elaboration of their knowledge bases and the introduction of specialised terms and concepts, these will still have to be related to the common world knowledge.

A growing mass of work at present is focussed on making some use of the definitional component of a dictionary entry, where the dictionary itself is regarded as a knowledge base, albeit presented in a loose and not very structured fashion. Starting with the assumption that diction- 
ary definitions both employ and imply a taxonomy of genus terms, the ultimate goal is to relate natural language words to this underlying structure which relates together the defining concepts in the dictionary. There are many problems here, ranging from the arbitrariness of dictionary definitions to the distribution of a particular piece of data over a number of separate entries to the fundamental differences between dictionaries and encyclopaedias.

Underneath even the most detailed dictionary definition there is still a substantial amount of general knowledge about the world. Without it being formalised and clearly stated, no complete analysis of the descriptive texts typically found in a dictionary can be fully achieved. Still, current views on automatic natural language processing tend to agree that it is hard to pinpoint a boundary between the semantic knowledge that the use of a particular word (sense) implies and the expert background which prompts its use in a specific domain. While it would be unreasonable to expect to find any of the latter in a dictionary source there is sufficient evidence to indicate that most of the former-whether presented in terms of selectional restrictions, markers, formulae constructed from semantic primitives, frame-based structures, propositional systems with sortal information encoded in the form of meaning postulates and associated with predicate symbols, or by some other means-can be derived from a suitable dictionary. (Note, however that the aim of the CYC project at MCC is precisely to derive a formal model of general world knowledge down to some level of detail from an encyclopaedia source.)

The idea of using "what is behind words"-structured assignment to thesauri classes or looser deseriptions via natural language expressions-to perform functions such as word-sense assignment or lexical disambiguation is certainly not new. Recent proposals for using dictionary definitions to determine relevant context consolidate and extend the pioneering efforts of the Cambridge Language Research Unit of 30 years ago where the structure of the Roget's Thesaurus was applied as an MT interlingua and supported word sense disambiguation.

However, now we are in position to evaluate critically "what is behind words". One of the reasons for the renewed interest in words is the availability, on-line (in the form of machine readable dictionaries and encyclopaedias), of vast resources of information about words. We also have the technology to both process these resources and extract from them what is relevant to computer programs concerned with various natural language activities. Still, given the shallow world knowledge typically contained in dictionaries, perhaps a complete semantic component for a natural language processing system cannot be derived fully automatically by suitable analysis of word definitions. The meaning content of a dictionary holds a promise of a different nature.

While there is no consensus on the kind of representation scheme best suited for capturing the knowledge required for language interpretation and understanding, it is nonetheless possible to identify the distinct classes of propositional systems and type-hierarchy systems. The latter utilise, broadly speaking, general notions of frame-like concepts with slot-like role descriptions, organised in an inheritance hierarchy along generalisation / specialisation axes. Most of the recent work on knowledge representation, whether representative of the strict type-hierarchy approach (exemplified by, e.g. FRL, KRL, NETL, UNITS, KL-ONE) or of the hybrid style of KRYPTON and KL-TWO, for example, can be cast into this general mould.

For natural language processing, where general world knowledge is just as important as specialised domain- and task-dependent knowledge, the utility of hierarchically structured net- 
works of concepts need not be emphasised. And while it is not entirely clear whether all of the structured information required by the system functions can be derived in a systematic and consistent way from dictionary sources, such sources offer a particularly good and convenient starting point for initial compilation of taxonomically structured knowledge about the world.

Taxonomies for existing dictionaries have been constructed, albeit in a semi-automatic way; more recently, fully automated procedures have been developed to accomplish the same task, and further elicitation of taxonomic structures is underway. Typically, natural language processing techniques and constraints from linguistic theory are applied to the word definitions in the dictionary; in some cases, the analysis process can be made sufficiently elaborate to accomplish not only the identification (and disambiguation) of the superordinate (genus) term, but also further elaboration of the defining concept by extracting additional modifiers and predications.

The resulting frame-like structures with filled-in slots, assigned defaults and specified constraints (insofar as these have been given in the original word definition) allow a fairly accurate characterisation of a concept both in a particular domain of discourse and within the relevant fragment of the hierarchy. The networks thus defined can be of enormous utility to a wide range of text and language processing applications.

Still, a much more important consequence of the assumption for an underlying taxonomy and the techniques developed for extracting such a taxonomy is the interesting prospect of looking at taxonomic organisations across a variety of existing dictionaries. Perhaps there is a common structure underneath definitions from different sources? Perhaps this common structure reflects a common world representation? It is too much to expect to find the "right" dictionary, which employs a sufficiently detailed and analytically designed taxonomy, directly utilisable by a natural language processing system. On the other hand, it would be unwise to ignore commonalities in the definitional spaces of a cross-section of dictionaries.

From a slightly different perspective, the whole body of work which looks at word definitions in dictionaries bears directly on the issue of deriving lexicons for language processing. Clearly, the techniques referred to above can be applied directly to the task of compiling lexical semantic information from dictionary sources. It turns out that these embody fairly detailed and complex linguistic and general world distinctions. Attempting to use individual word definitions to derive, semi-automatically, semantic formulae which incorporate selectional restrictions and shallow world knowledge is not an isolated activity. Still, as already noted, dictionaries are not complete repositories of linguistic and world knowledge, and it is yet not clear whether a direct mapping from a word definition to a lexical entry, derived by means indicated here, is going to be an entirely profitable exercise.

In order to exploit fully the wealth of linguistic and general world knowledge embodied in a dictionary, we need to look at the old issue of semantic primitives from a different perspective. It has already been suggested that fixed sets of primitive concepts cannot cope as the granularity of different domains and contexts varies. A lot of questions have also been raised by the issue of exactly how to arrive at a particular set of primitives. It would seem that the work currently being done on analysing the taxonomic organisations underlying a number of existing dictionaries offers a solution here. 
At least one dictionary source has been produced by adhering to a principle of using a limited core vocabulary of basic words, only in their central meanings, for all the definitions; this controlled vocabulary clearly has not been selected randomly. Apart from allowing a fairly close tuning of a definitions analysis program (it turns out that there exists, in effect, a grammar for writing word definitions), the application of such a program to the core vocabulary itself will yield the taxonomy of defining concepts used in the process of the dictionary writing. Taking this as a guideline for determining a set of semantic primitives, individual word definitions can then be analysed and corresponding semantic formulae compiled within this primitive vocabulary.

Perhaps the most pertinent point here is that neither this, nor any other dictionary has been developed with the particular intention of being used by a natural language processing program. Given that we now seem to have a slightly better idea of what we would like our systems to do, that a growing collection of texts, from which significant lexical information can be derived, exists in machine readable form, and that we know enough about parsing, a question which would be appropriate to ask is this: what can "reverse engineering" of a dictionary do for a knowledgeintensive language processing system?

\section{REFERENCES}

Alshawi,H. (1986) Processing Dictionary Definitions with Phrasal Pattern Hierarchies. Computer Laboratory, University of Cambridge.

Amsler,R. (1980) The Structure of the Merriam-Webster Pocket Dictionary. Doctoral dissertation, TR-164, Computer Science Department, University of Texas, Austin.

Boguraev,B. (1986) Machine-readable dictionaries and research in computational linguistics. Proceedings of a Workshop on Automating the Lexicon, Grosseto, Italy.

Calzolari,N. (i984) Detecting patterns in a lexical database. Tenth International Congress on Computational Linguistics, Stanford, California, 2-6 July 1984. Association for Computational Linguistics, Bell Communications Research, pp. 170-173.

Chodorow,M.; Byrd,R.; and Heidorn,G. (1985) Extracting semantic hierarchies from a large online dictionary. Proceedings, 23rd Annual Meeting of the Association for Computational Linguistics, Chicago, Illinois, 8-12 July 1985. Association for Computational Linguistics, Bell Communications Research, pp. 299-304.

Lenat,D.; Prakash,M.; and Shepherd,M. (1986) CYC: Using common sense knowledge to overcome brittleness and knowledge acquisition bottlenecks. AI Magazine, 6(4), 65-92.

Lesk,M. (1986) Automatic sense disambiguation: how to tell a pine cone from an ice cream cone. Bell Communications Research, Morristown, New Jersey.

Masterman,M.; Needham, R.M.; Sparck Jones,K.; and Mayoh,B. (1957) "AGRICOLA INCURVO TERRAM DIMOVIT ARATRO". First stage translation into English with the aid of Roget's Thesaurus. Report (ML84) ML92, Cambridge Language Research Unit, Cambridge, England. 
Wilks, Y. (1977) Good and bad arguments about semantic primitives. Communication and Cognition, 10, 181-221. 